\title{
Effects of temperature, pressure, and carrier gases on the performance of an aerosol particle mass analyser
}

\author{
Ta-Chih Hsiao ${ }^{1,3}$, Li-Hao Young ${ }^{2}$, Yu-Chun Tai ${ }^{3}$, and Po-Kai Chang ${ }^{1}$ \\ ${ }^{1}$ Graduate Institute of Environmental Engineering, National Taiwan University, Taipei, 10673, Taiwan \\ ${ }^{2}$ Department of Occupational Safety and Health, China Medical University, Taichung, 40402, Taiwan \\ ${ }^{3}$ Graduate Institute of Environmental Engineering, National Central University, Taoyuan, 32001, Taiwan
}

Correspondence: Ta-Chih Hsiao (tchsiao@gmail.com)

Received: 23 December 2017 - Discussion started: 3 April 2018

Revised: 25 June 2018 - Accepted: 13 July 2018 - Published: 9 August 2018

\begin{abstract}
Effective density is a crucial parameter used to predict the transport behaviour and fate of particles in the atmosphere, and to measure instruments used ultimately in the human respiratory tract (Ristimäki et al., 2002). The aerosol particle mass analyser (APM) was first proposed by Ehara et al. (1996) and is used to determine the effective density of aerosol particles. A compact design (Kanomax APM3601) was subsequently developed by Tajima et al. (2013). Recently, a growing number of field studies have reported application of the APM, and experimental schemes using the differential mobility analyser alongside the APM have been adopted extensively. However, environmental conditions such as ambient pressure and temperature vary with the experimental location, and this could affect the performance of the APM. Gas viscosity and Cunningham slip factors are parameters associated with temperature and pressure and are included in the APM's classification performance parameter: $\lambda$. In this study, the effects of temperature and pressure were analysed through theoretical calculation, and the influence of varying carrier gas was experimentally evaluated. The transfer function and APM operational region were further calculated and discussed to examine their applicability. Based on the theoretical analysis of the APM's operational region, the mass detection limits are changed with the properties of carrier gases under a chosen $\lambda$ value. Moreover, the detection limit can be lowered when the pressure is reduced, which implies that performance may be affected during field study. In experimental evaluation, air, oxygen, and carbon dioxide were selected to atomize aerosols in the laboratory with the aim of evaluating the effect of gas viscosity on the APM's performance. Using monodisperse polystyrene latex (PSL)
\end{abstract}

spheres with nominal diameters of 50 and $100 \mathrm{~nm}$, the classification performance of the APM was slightly varied with carrier gases, while the classification accuracy was consistently within $10 \%$.

\section{Introduction}

To determine the adverse health effects of inhalable particles, the lung regional deposition fraction must be investigated (Chuang et al., 2016; Haddrell et al., 2015). The lung regional deposition fraction is closely associated with the density and morphology of submicron particles that can be derived from their mass measurement (Broday and Rosenzweig, 2011; Salma et al., 2002; Shi et al., 2015). Therefore, mass distribution and particle density play pivotal roles in the study of associated health effects. However, obtaining measurements of the morphology or density of aerosol particles in the environment is not easy (Bau et al., 2014; DeCarlo et al., 2004), and particles in an ambient environment are generally irregular and nonspherical. In this regard, Liu et al. (2013) demonstrated that the morphological parameter of a fractal soot particle, namely the fractal dimension $\left(D_{\mathrm{f}}\right)$, could affect the particle's radiative properties. In addition, according to theoretical calculations, He et al. (2015) reported that aged soot aggregates with partially encapsulated or externally attached structures have weaker absorption properties than do fresh soot aggregates.

Nevertheless, theoretical simulations and experimental studies on the effects of aerosols on human health and the atmosphere all require certain assumptions regarding parti- 
cle morphology or density to be made to enable the conversion of number concentration to mass concentration or volume concentration (Hand and Kreidenweis, 2002). To experimentally retrieve the density of submicron-to-nanometresized particles, a differential mobility analyser (DMA) system coupled with a low-pressure impactor was developed to measure the aerodynamic sizes of particles of known electric mobility size. By further assuming that the particles were all spherical, the "effective" or "apparent" density was derived (Kelly and McMurry, 1992; Schleicher et al., 1995; Skillas et al., 1998). A similar system combining a scanning mobility particle sizer and electrical low-pressure impactor in parallel was reported and applied to study diesel particulate matter and ambient aerosols (Maricq et al., 2000; Ristimäki et al., 2002; Symonds et al., 2007; Van Gulijk et al., 2004; Virtanen et al., 2004). Furthermore, Ehara et al. (1996) developed the aerosol particle mass analyser (APM), which is an aerosol instrument that classifies particle mass by balancing the centrifugal force and electrostatic force. Based on an identical classification mechanism but utilizing a different rotating scheme to create centrifugal force, the Couette centrifugal particle mass analyser is another commercially available instrument. With such advances in aerosol instrumentation, McMurray et al. (2002) proposed using the APM to directly measure the mass of monodispersed particles classified by a DMA (tandem DMA-APM system) instead of using an impactor to probe the particle aerodynamic size. This DMAAPM scheme is capable of revealing the density or mass distribution of targeted aerosol particles in real time. Throughout the past decades, this scheme has also been adopted extensively to determine the $D_{\mathrm{f}}$ of aerosol aggregates (Lall et al., 2008; McMurry et al., 2002; Park et al., 2003, 2004a, b; Scheckman et al., 2009) and atmospheric aerosols (Kuwata and Kondo, 2009; Kuwata et al., 2011).

A growing number of APM experiments are being conducted in outdoor environments in relation to the dual interest in human health and climate change (Leskinen et al., 2014; Rissler et al., 2014). However, when experiments are conducted in the field, environmental conditions such as temperature $(T)$ and pressure $(P)$ vary spatially and temporally, particularly at high-altitude sites. The viscosity $(\mu=$ $\left.\mu_{r}\left(\frac{T_{r}+S_{u}}{T+S_{u}}\right)\left(\frac{T}{T_{r}}\right)^{1.5}\right)$ and mean free path $\left(l=\frac{\mu}{P} \sqrt{\frac{\pi R T}{2 M}}=\right.$ $l_{r}\left(\frac{P_{r}}{P}\right)\left(\frac{T}{T_{r}}\right)^{2}\left(\frac{T_{r}+S_{u}}{T+S_{u}}\right)$ ) of ambient air change continuously (Kulkarni et al., 2011), thereby influencing the performance and range of detection limits (classifiable region) for the APM. Therefore, in this study, air, oxygen, and carbon dioxide were selected as carrier gases to experimentally evaluate the effect of gas viscosity and the mean free path on the performance of the APM, including the classifiable region and detection limits. On the other hand, argon would be required as the carrier gas if the APM were used as an aerosol particle classifier coupled with inductively coupled plasma mass spectrometry (ICP-MS; in a similar manner to the DMA-
ICP-MS system) (Myojo et al., 2002). Therefore, the effects of changing the carrier gas in the APM's transfer function require investigation. In this regard, the experimental and simulated transfer functions for the APM operated for various carrier gases were explored and are compared in this paper. The results provide a valuable insight into the performance of the APM when operated under various conditions.

\section{Operational theory}

The APM was first proposed by Ehara et al. (1996), and a compact version was recently developed by Tajima et al. (2013). The instrument consists of two electrodes, namely coaxial and rotating cylindrical electrodes, between which a narrow annular space is created for mass classification (classification zone). When the APM is initialized, the inner and outer electrodes rotate at the same speed $(\omega)$ to generate centrifugal force, and a high voltage is applied to the inner electrode to create a "counter" electrostatic force. The governing equations of particle movements in radial and axial directions inside the classification zone are expressed as follows (Ehara et al., 1996):

$$
\begin{aligned}
& \frac{m}{\tau} \cdot \frac{\mathrm{d} r}{\mathrm{~d} t}=m \cdot r \cdot \omega^{2}-\frac{q \cdot V}{r \cdot \ln \left(r_{o} / r_{i}\right)} \text { (in a radial direction), } \\
& \frac{m}{\tau}\left\{\frac{\mathrm{d} z}{\mathrm{~d} t}-v(r)\right\}=0 \quad \text { (in an axial direction), } \\
& \tau=\frac{\rho_{p} \cdot d_{p, m}^{2} \cdot C_{\mathrm{c}}\left(d_{p, m}\right)}{18 \mu}, \\
& S_{\mathrm{c}}=\frac{m}{q}=\frac{V}{r_{\mathrm{c}}^{2} \cdot \omega^{2} \cdot \ln \left(r_{0} / r_{i}\right)} .
\end{aligned}
$$

Particles are then classified based on the balance between the centrifugal force and counter electrostatic force, and only particles with a designated mass-to-charge ratio $(S)$ pass through the classification zone. The mass-to-charge ratio for particles that remain in the central line $\left(r_{\mathrm{c}}\right)$ within the classification zone is defined as the "critical" $S\left(S_{\mathrm{c}}\right.$; Eq. (4). Based on this definition, the classified particle mass $(m)$, rotation speed $(\omega)$, and voltage applied $(V)$ are linearly correlated on a log scale, as shown in Eq. (5):

$$
\begin{aligned}
\log (m) & =\left[\log (q)-2 \log \left(r_{\mathrm{c}}\right)-\log \left(\ln \left(r_{0} / r_{i}\right)\right)\right] \\
& +\log (V)-2 \log (\omega) .
\end{aligned}
$$

Tajima et al. $(2011,2013)$ depicted the classifiable region for the APM by using a log-log mass-versus-rotation-speed plot. However, because of the physical limits of the operational voltage and rotation speed of the APM, a parallelogram of potential classifiable regions was reported.

The transfer function $(\Omega)$ is generally used to characterize the classification performance of the DMA, and by defining the penetration probability of particles under a designed condition, the transfer function is employed to evaluate the performance of the APM. The nondiffusive transfer function for 
the APM $\left(\Omega_{\mathrm{APM}}\right)$ can be indexed by the penetration at the $S_{\mathrm{c}}\left(t\left(S_{\mathrm{c}}\right)\right)$ and the resolution parameter $(\Delta S)$ (Tajima et al., $2013) ; t\left(S_{\mathrm{c}}\right)$ is the maximum height of the transfer function, and $\Delta S$ can be estimated theoretically as follows:

$$
\begin{aligned}
& t\left(S_{\mathrm{c}}\right)=\exp \left(-\lambda_{\mathrm{c}}\right), \\
& \Delta S=\frac{r_{\mathrm{c}}}{4 \delta} \tanh \left(\lambda_{\mathrm{c}} / 2\right) .
\end{aligned}
$$

Both parameters are closely related to $\lambda_{c}$, which is the major dimensionless performance parameter defined by Ehara et al. (1996) for APM operation. The $\Omega_{\mathrm{APM}}$ is a strong function of $\lambda_{c}$ :

$$
\begin{aligned}
\lambda_{\mathrm{c}} & =\frac{2 \tau \cdot \omega^{2} \cdot L}{\bar{v}}=\frac{2 \cdot \frac{\rho_{p} \cdot d_{p, m}^{2}}{18} \cdot \omega^{2} \cdot L}{\left(Q_{a} / \pi\left(r_{o}^{2}-r_{i}^{2}\right)\right)} \cdot \frac{C_{\mathrm{c}}\left(d_{p, m}\right)}{\mu} \\
& =\frac{2 m 3 d_{p, m} \cdot \omega^{2} \cdot L}{\left(Q_{a} /\left(r_{o}^{2}-r_{i}^{2}\right)\right)} \cdot \frac{C_{\mathrm{c}}\left(d_{p, m}\right)}{\mu} .
\end{aligned}
$$

$\lambda_{c}$ can be interpreted as the ratio of the timescale for axial and radial particle movements in the classification zone. When the value of $\lambda_{c}$ is larger, the APM has a superior classification resolution. Generally, the axial traversal timescale $(L / \bar{v})$ is considered constant because the aerosol flow rate is fixed when the instrument is operated. Thus, the classification performance can be improved by decreasing the radial traversal timescale $\left(1 / 2 \tau \cdot \omega^{2}\right)$. A shorter radial traversal time enables easier removal of incompetent particles by deposition onto the electrodes, which denotes lower penetration possibility for particles with a mass-to-charge ratio other than $S_{\text {c }}$. In other words, the transfer function is narrower, or $\Delta S$ is smaller, for a larger value of $\lambda_{c}$, which suggests a higher classifying resolution. However, the trade-off for operating the APM at a larger $\lambda_{\mathrm{c}}$ is that the maximum penetration of the transfer function $\left(t\left(S_{\mathrm{c}}\right)\right)$ is lower. Tajima et al. (2011) recommended that the APM be operated at a constant $\lambda_{\mathrm{c}}$ (with a fixed $\Omega_{\text {APM }}$ ) within the range of 0.25 to 0.5 when the aerosol flow rate is $0.3 \mathrm{~L} \mathrm{~min}^{-1}$.

For a constant value of $\lambda_{c}$, a unique curve in the loglog mass-versus-rotation-speed plot can be determined iteratively through Eq. (8), and this intercepts the APM classifiable region. This constant- $\lambda_{c}$ curve generally acts as the lower boundary for the APM classifiable region. As observed in Eq. (8), $\lambda_{c}$ is a function of the APM rotation speed $(\omega)$ and depends on particle mass and density with respect to relaxation time $(\tau)$. The value of $\tau$ is closely related to gas viscosity $(\mu)$ and the Cunningham slip factor $\left(C_{\mathrm{c}}\right)$. At a given $\lambda_{\mathrm{c}}$, the $\omega$ for classifying particles with a certain mass decreases with an increasing $C_{\mathrm{c}}$-to- $\mu$ ratio $\left(\frac{C_{\mathrm{c}}}{\mu}\right)$. For air, oxygen, argon, and carbon dioxide, limited variations in the values of $\frac{C_{\mathrm{c}}}{\mu}$ are observed, and their constant- $\lambda_{\mathrm{c}}$ curves are closely clustered. By contrast, the long mean free paths of low-molecularweight gases such as hydrogen and helium can lead to a relatively high $\frac{C_{\mathrm{c}}}{\mu}$. Consequently, when the APM is operated with

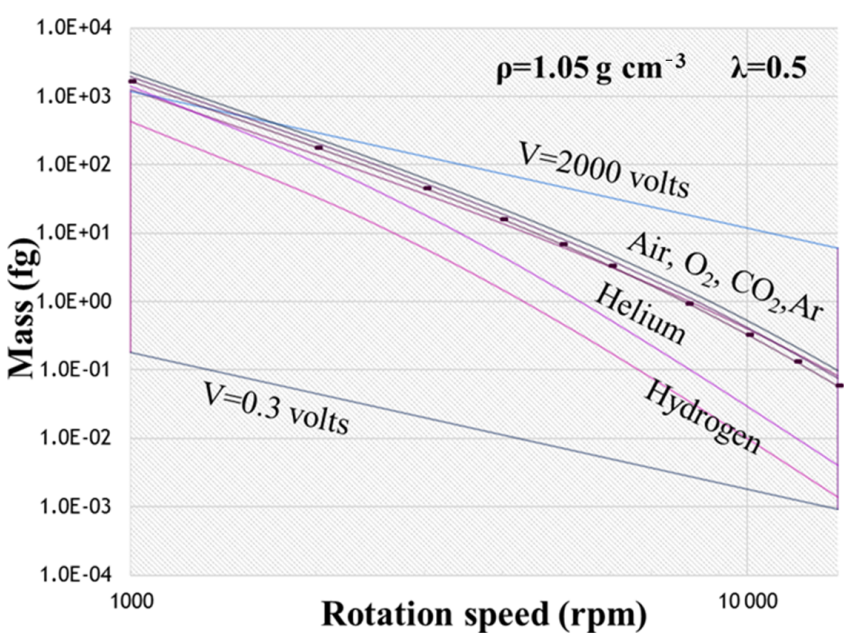

Figure 1. Operational region of APM.

hydrogen and helium, the classifiable mass range needs to be extended to a much lower detection limit without sacrificing the resolution or modifying the APM hardware (Fig. 1). However, an implicit problem is that the breakdown voltages of inert gases are generally approximately 1 order of magnitude lower than that of air (Schmid et al., 2002) (values of gas-specific parameters and the corresponding detection limits are listed in Table 1).

The expression of $\frac{C_{\mathrm{c}}}{\mu}$ can be further rewritten to reveal the effects of temperature, pressure, and the carrier gas species as Eq. (9):

$$
\begin{aligned}
\frac{C_{\mathrm{c}}\left(d_{p}\right)}{\mu} & =\frac{1}{\mu}+\frac{2 l / d_{p}}{\mu}\left[\alpha+\beta \cdot \exp \left(\frac{-\gamma}{2 l / d_{p}}\right)\right] \\
& =\left(\frac{T+S_{u}}{T_{r}+S_{u}}\right)\left(\frac{T}{T_{r}}\right)^{-1.5} \cdot \frac{1}{\mu_{r}}+\left(\frac{P_{r}}{P}\right)\left(\frac{T}{T_{r}}\right)^{0.5} \\
& \cdot \frac{2 l_{r} / d_{p}}{\mu_{r}}\left[\alpha+\beta \cdot \exp \left(\left(\frac{P}{P_{r}}\right)\left(\frac{T}{T_{r}}\right)^{-2}\left(\frac{T+S_{u}}{T_{r}+S_{u}}\right)\right.\right. \\
& \left.\left.\cdot\left(\frac{-\gamma}{2 l_{r} / d_{p}}\right)\right)\right]
\end{aligned}
$$

where $\alpha=1.142, \beta=0.558$, and $\gamma=0.999$ (Allen and Raabe, 1985). As shown in Fig. 2, compared to pressure, $\frac{C_{\mathrm{c}}}{\mu}$ is somewhat invariant with temperature; when the temperature changes from 273 to $318 \mathrm{~K}$, the difference of $\frac{C_{\mathrm{c}}}{\mu}$ for all gas species is less than $6 \%$. However, the $\frac{C_{\mathrm{c}}}{\mu}$ for $100 \mathrm{~nm}$ particles increases by $42 \%$ when the air pressure decreases to $65 \mathrm{kPa}$ (Fig. 3); therefore, when operating the APM at highaltitude sites, the detection limits need to be lowered or the resolution needs to be improved. By contrast, based on the analytical predictions, Eq. (4) is unaffected by $\frac{C_{\mathrm{c}}}{\mu}$, and the classification accuracy remains unchanged. 
Table 1. Properties of gases at normal temperature and pressure (Kulkarni et al., 2011) and corresponding APM detection limits at $\lambda=0.5$.

\begin{tabular}{lrrrrrrrr}
\hline Gas & $\begin{array}{r}M \\
\left(\mathrm{~g} \mathrm{~mol}^{-1}\right)\end{array}$ & $\begin{array}{r}\rho \\
\left(\mathrm{Kg} \mathrm{m}^{-3}\right)\end{array}$ & $\begin{array}{r}S_{u} \\
(\mathrm{~K})\end{array}$ & $\begin{array}{r}\mu_{r} \\
\left(10^{-6} \mathrm{~N} \mathrm{~s} \mathrm{~m}^{-2}\right)\end{array}$ & $\begin{array}{r}\ell_{r} \\
(\mathrm{~nm})\end{array}$ & $\begin{array}{r}d_{p, m}^{*} \\
(\mathrm{~nm})\end{array}$ & $\begin{array}{r}\text { Min. mass } \\
\left(10^{-2} \mathrm{fg}\right)\end{array}$ & $\begin{array}{r}\text { Max. mass } \\
\left(10^{-2} \mathrm{fg}\right)\end{array}$ \\
\hline $\mathrm{CO}_{2}$ & 44.010 & 1.842 & 220.5 & 14.673 & 43.2 & $53.3-1316.9$ & 8.3 & 1.3 \\
$\mathrm{Air}$ & 28.966 & 1.205 & 110.4 & 18.203 & 66.5 & $47.5-1448.8$ & 5.9 & 1.7 \\
$\mathrm{O}_{2}$ & 32.000 & 1.331 & 116.8 & 20.229 & 69.1 & $51.8-1524.8$ & 7.6 & 1.9 \\
$\mathrm{Ar}$ & 39.948 & 1.661 & 141.4 & 22.292 & 69.4 & $56.4-1608.3$ & 9.9 & 2.3 \\
$\mathrm{H}_{2}$ & 2.016 & 0.090 & 66.7 & 8.799 & 123 & $13.6-921.3$ & 0.14 & 0.43 \\
$\mathrm{He}$ & 4.003 & 0.166 & 73.8 & 19.571 & 192 & $19.5-1365.0$ & 0.41 & 1.4 \\
\hline
\end{tabular}

$* d_{p, m}$ detection limits were calculated assuming that $\rho=1.05 \mathrm{~g} \mathrm{~cm}^{-3}$.

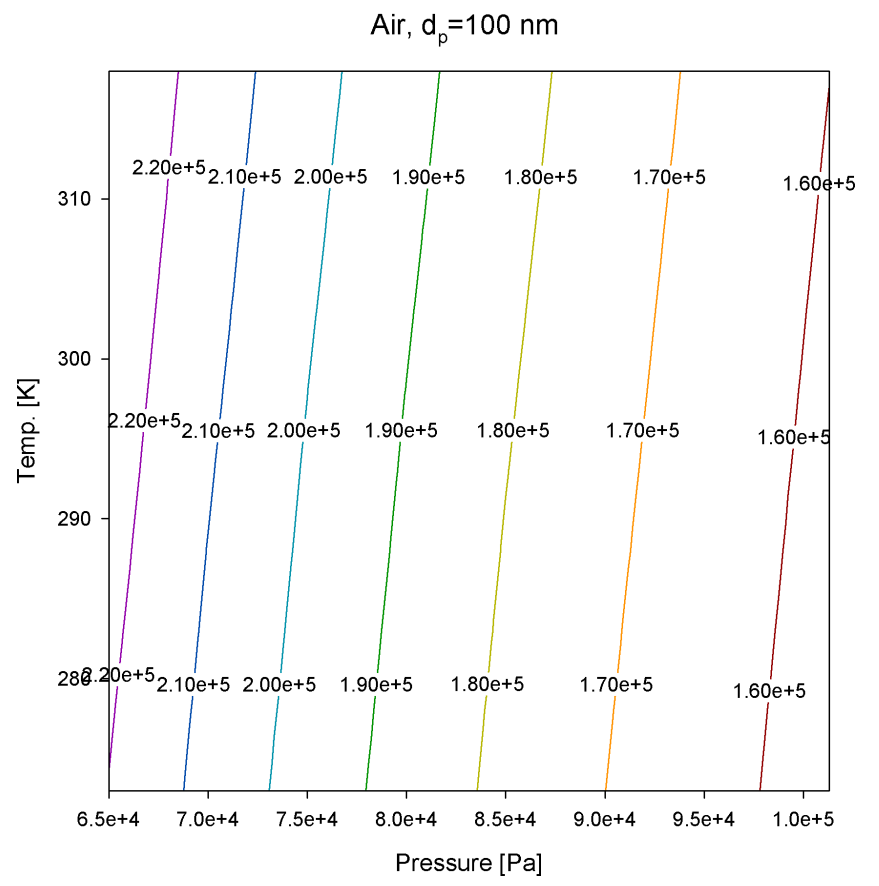

Figure 2. $\frac{C_{\mathrm{c}}}{\mu}$ ratio for various temperatures and pressures.

\section{Experimental method}

Figure 4 depicts the experimental evaluation system. The particles were generated by an aerosol atomizer (TSI, Model 3076) and dehumidified by two desiccant dyers connected in series to remove excess water content. To experimentally evaluate the classification accuracy, 50 and $100 \mathrm{~nm}$ polystyrene latex (PSL) spheres certified by the National Institute of Standards and Technology (Thermo Fisher Scientific, cat. no. 3050A and 3100A) were used here. The mean diameters of the size distributions of the 50 and $100 \mathrm{~nm}$ PSL given by the manufacturers are $46 \pm 2$ and $100 \pm 3 \mathrm{~nm}$, respectively. These PSL particles were classified using the DMA (TSI 3081) and then delivered to the APM (Kanomax model II-3601) to determine particle mass. The overall aerosol flow rate of the DMA-APM system was controlled by the down-

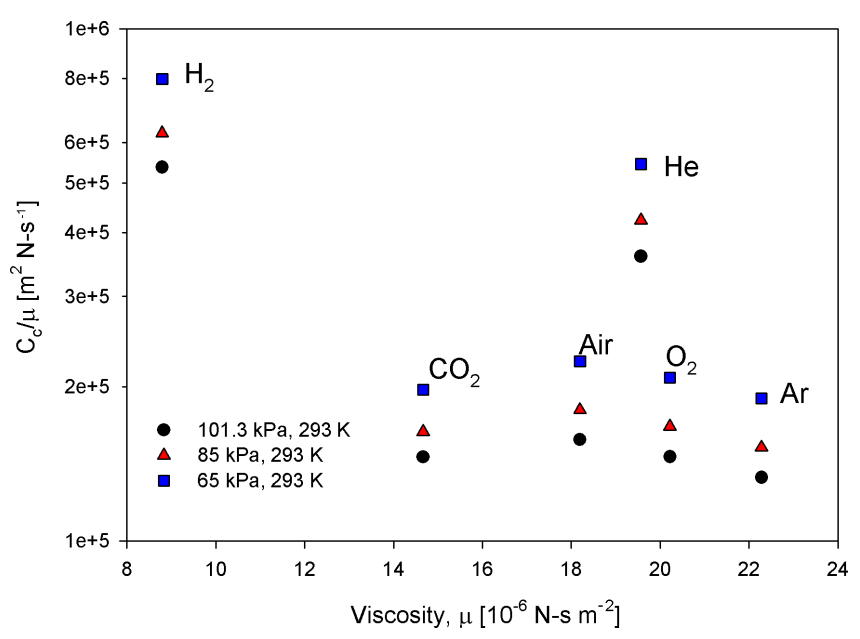

Figure 3. Pressure effect on $C_{\mathrm{c}}$-to- $\mu$ ratio for various carrier gases.

stream CPC (condensation particle counter, TSI 3022A) and fixed at $0.3 \mathrm{~L} \mathrm{~min}^{-1}$. To optimize the size of the classification resolution, the sheath flow rate of the DMA was set at 3.0 $\mathrm{L} \mathrm{min}^{-1}$. In addition to air, carbon dioxide $\left(\mathrm{CO}_{2}\right)$ and oxygen $\left(\mathrm{O}_{2}\right)$ with purity levels of $99.99 \%$ were supplied as carrier gases. Before conducting measurements, the selected gas was used to purge all apparatus for at least 5 min to ensure that no residual contaminant gases remained, and the flow rates of the DMA's sheath flow and aerosol flow were calibrated using a volumetric flow meter (Gilian Gilibrator 2, Sensidyne, St. Petersburg, FL, USA). Because the DMAAPM was used to investigate the effects of carrier gases on the APM's performance, it was necessary to first study the sizing accuracy of the DMA for various carrier gases. The results showed that the differences between the measured modal diameters and nominal sizes of 50 and $100 \mathrm{~nm}$ certified PSL particles were within $6 \%$ for air, $\mathrm{CO}_{2}$, and $\mathrm{O}_{2}$. Therefore, no significant effects of gas species on DMA sizing accuracy were observed, which was consistent with the findings of Schmid et al. (2002).

The classifying accuracy of aerosol instruments under various conditions is generally characterized by a normalized indicator relative to a known reference (Karg et al., 1992; 


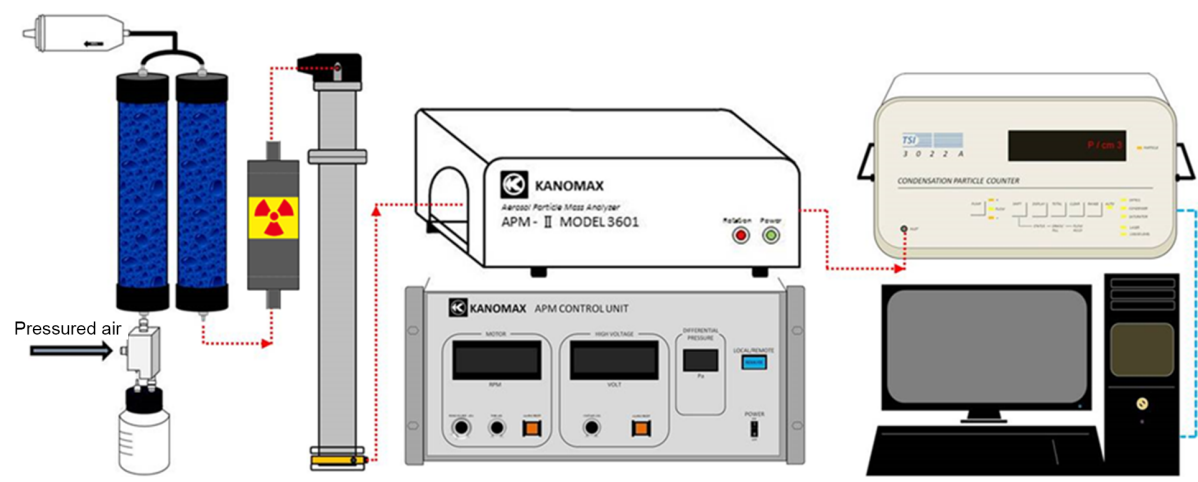

Figure 4. Experimental DMA-APM system.

Marlow et al., 1976; Ogren, 1980; Schmid et al., 2002). The normalized mass-to-charge ratio $\left(\bar{S}=\frac{S_{\text {gas }}}{S_{\text {air }}}\right)$ was used in the present study to analyse the APM's performance at a constant rotation speed $(\omega)$ and constant sizing resolution $(\lambda)$ operation. For the constant $\lambda$ operation, $\lambda$ values of 0.24 and 0.45 were chosen for both 50 and $100 \mathrm{~nm}$ PSL spheres. The operation of DMA-APM is identical to Kuwata and Kondo (2009) and Kuwata et al. (2011), in which the DMA selects particles with +1 charge and predetermined mobility diameters and then subjects them to the APM. Subsequently, the APM was set to scan across a range of voltage $(V)$, while the number concentration $\left(C_{\mathrm{N}}\right)$ of the passing particles was measured by a CPC. The peak of the $C_{\mathrm{N}}-V$ distribution was subsequently inspected to determine the particle mass $(m)$.

The APM's stepping $C_{\mathrm{N}}-V$ spectrum was converted to the transfer function and compared with the simulated transfer function. The transfer function is a kernel function used to theoretically evaluate the concentration of downstream particles and is critical for evaluating the performance of the APM (Ehara et al., 1996; Emery, 2005; Tajima et al., 2013). In the present study, two computer programs, namely the TRANSFER program and SIM_APM program (developed by the National Institute of Advanced Science and Technology (AIST) of Japan) were utilized to theoretically evaluate the operational performance of the APM (Tajima et al., 2011, 2013). The TRANSFER program calculated the theoretical APM transfer function at a fixed rotation speed and voltage, and the SIM_APM program simulated particle distribution at the APM outlet based on the known particle distribution at the APM inlet (the size distribution was classified by the front DMA). Both simulated results were compared with experimental APM measurements.

\section{Results and discussion}

\subsection{Constant $\lambda$}

For the case of constant $\lambda$ operation, $\bar{S}$ is expressed as Eq. (10), where $\bar{S}$ is mainly a function of the voltage applied to the $\operatorname{APM}(V)$, Cunningham slip correction factor $\left(C_{\mathrm{c}}\right)$, and gas viscosity $(\mu)$ :

$$
\bar{S}=\frac{S_{\mathrm{gas}}}{S_{\mathrm{air}}}=\frac{V_{\mathrm{gas}}}{V_{\mathrm{air}}} \frac{C_{\mathrm{c}, \text { gas }} / \mu_{\mathrm{gas}}}{C_{\mathrm{c}, \mathrm{air}} / \mu_{\mathrm{air}}} .
$$

To consider the various properties of the gas species, $\omega$ was adjusted to enable $\lambda$ to be fixed at approximately 0.24 and 0.45 for $50 \mathrm{~nm}$ PSL particles, as shown in Table 2. The results revealed that particle mass was generally underestimated for cases where $\mathrm{CO}_{2}$ was used as a carrier gas. In particular, underestimation was $23-25 \%$ for a $50 \mathrm{~nm}$ PSL sphere. By contrast, when $\mathrm{O}_{2}$ was used as the carrier gas, an overestimation of mass measurements was observed, with an error within $9 \%$.

As reported by Lall et al. $(2009,2008)$, the particle concentration measured as a function of APM voltage is wider than the APM transfer function even though the particle can be considered as "monodisperse" in size. This is mainly due the spread in calibration particle sizes or the transfer function of the DMA. To further eliminate the spread propagated from DMA classification, the transfer function of the APM was calculated using software developed by the AIST of Japan. The transfer function predicted (hereafter referred to as "predicted $\Omega_{\mathrm{APM}}$ ") based on the known size distribution of the DMA outlet (convoluted with the known size distribution classified by DMA) is indicated by the blue line in Fig. 6, and the theoretical transfer function (hereafter referred to as "theoretical $\Omega_{\mathrm{APM}}$ ") at a fixed optimal experimental peak voltage is indicated by a thinner green line. Experimental data points are indicated by the dotted symbol in Fig. 6. It should be noted that, according to the work done by Kuwata (2015), even when the resolution of the DMA-APM system appears to be controlled by the APM, the particle classification by the DMA-APM at a certain operating condition still could be regulated by both DMA and APM.

The APM resolution defined as $s_{\mathrm{c}} / \Delta s$ is used as an indicator to evaluate the classification performance. The resolution is recommended to be between 0.5 and 1.2 when interpreted as $\Delta s / s_{\mathrm{c}}$ (Emery, 2005; Tajima et al., 2011). However, in 


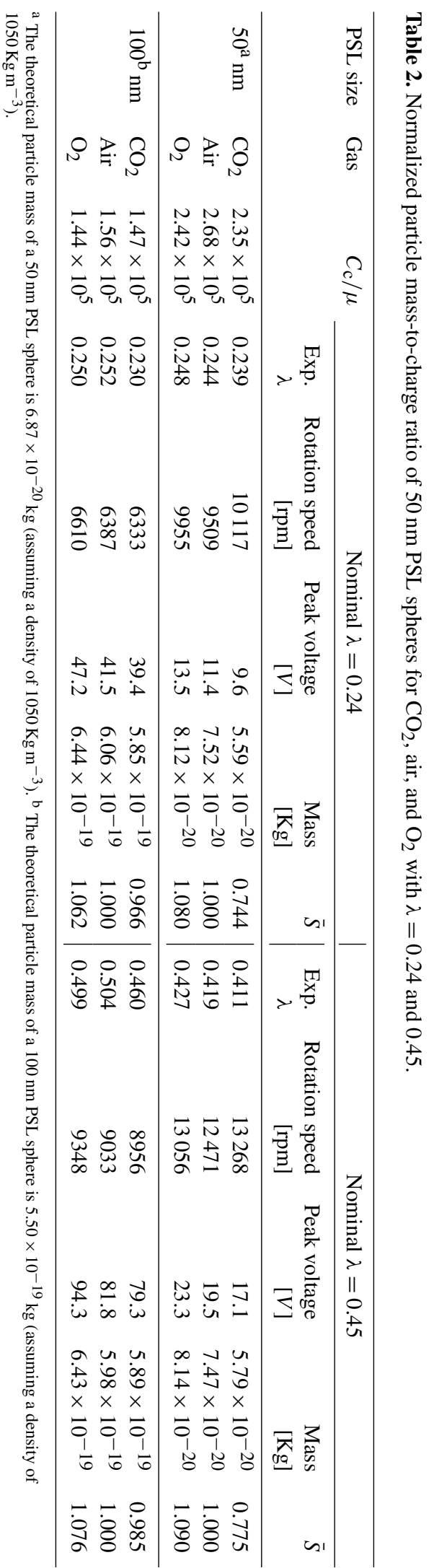

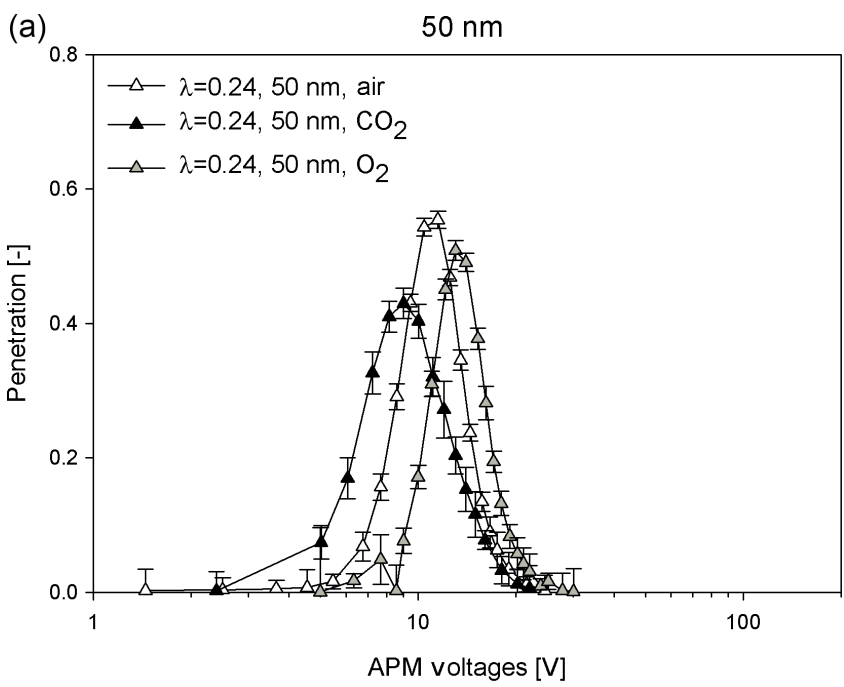

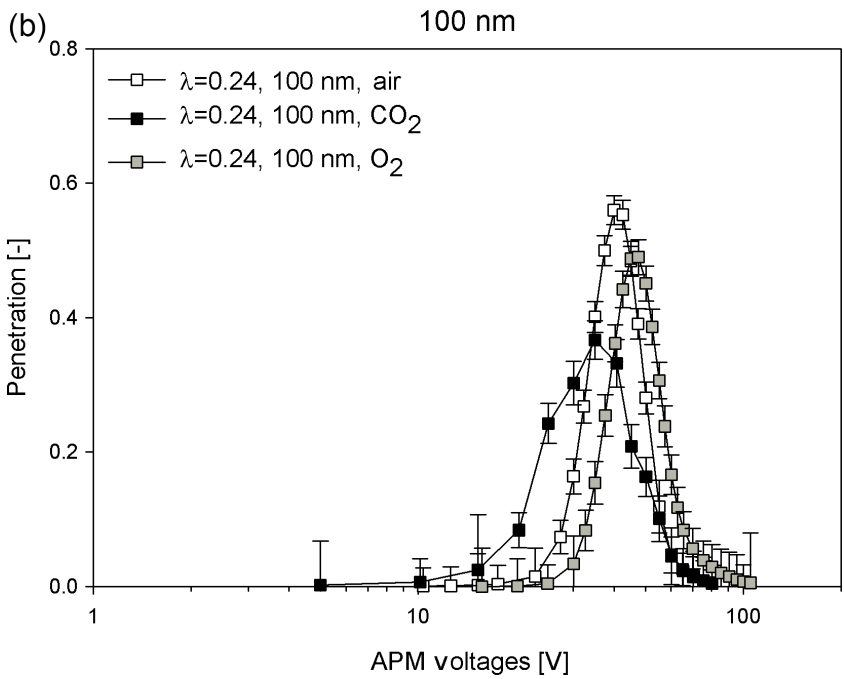

Figure 5. APM experimental transfer function of 50 and $100 \mathrm{~nm}$ PSL spheres for various carrier gases.

this study, the values of $s_{\mathrm{c}} / \Delta s$ for $\mathrm{CO}_{2}$ were 0.43 and 0.44 for 50 and $100 \mathrm{~nm}$ PSL spheres, respectively. Furthermore, the experimental optimal voltage in the case of $\mathrm{CO}_{2}$ was consistently lower than the theoretical voltage after convolution with the classified size distribution, and in the case of $\mathrm{O}_{2}$ it was slightly higher than the theoretical voltage. As shown in Table 1, the viscosity of $\mathrm{CO}_{2}$ was lower than that of air, whereas the viscosity of $\mathrm{O}_{2}$ was higher than that of air. These findings exhibit qualitative agreement with observations of under- or overestimations of PSL spheres. Therefore, we suspect that the fluid field in the APM classification zone is influenced by gas-specific properties such as $\mu$ and $\rho$. A further numerical simulation of the flow field was performed using COMSOL Multiphysics 4.3a. Using the flow velocity of air as a reference, the velocity differences in an angular direction at the APM's classification zone under various $\omega$ 

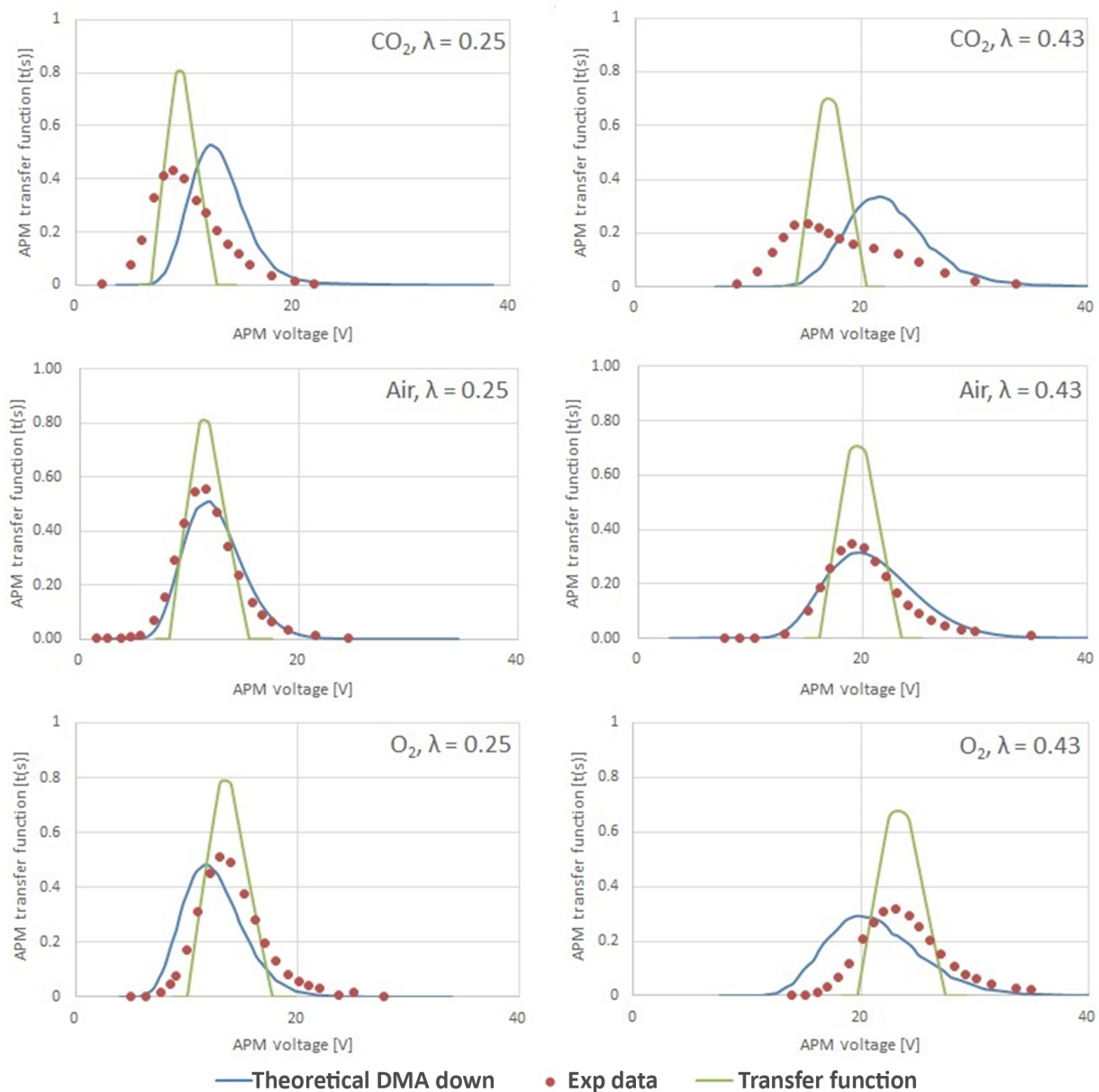

Figure 6. APM transfer function of $50 \mathrm{~nm}$ PSL spheres for various carrier gases with $\lambda=0.25$ and 0.43 .

values are plotted in Fig. 7. The velocity was generally lower in the classification zone of the APM when $\mathrm{CO}_{2}$ was used as the carrier gas, and an increase in the distinct differences between $\mathrm{CO}_{2}$ and air with an increase in the rotation speed was observed. Therefore, a lower viscosity and higher gas density likely intensify the shear force required to create rotating flow inside the APM. Because of the lower rotating flow velocity, significant deviations were observed in the measured results under normal conditions in the case of $\mathrm{CO}_{2}$; this phenomenon is intensified with higher values of $\omega$ and is more significant for small particles, which are even more prone to influence from the flow field.

\subsection{Constant $\omega$}

According to Kuwata's theoretical analysis of transfer function and resolution of the DMA-APM system, the common operation of constant $\omega$ and varying $V$ could not maintain the transfer function because of the range of $d_{p, m}$ passing the DMA (Kuwata, 2015). In such a case, the transfer function may not be symmetric, and the transfer function is narrower for larger $m$ because of the dependence of $\lambda_{c}$ on $m$. It was
Table 3. Normalized particle mass-to-charge ratio of $100 \mathrm{~nm}$ PSL spheres for $\mathrm{CO}_{2}$, air, and $\mathrm{O}_{2}$ ( $\omega$ is fixed at $\left.6375.5 \mathrm{rpm}\right)$.

\begin{tabular}{lrrr}
\hline $100 \mathrm{~nm}$ PSL & Peak voltage $[V]$ & Mass $[\mathrm{Kg}]$ & $\bar{S}$ \\
\hline $\mathrm{CO}_{2}, \lambda=0.22$ & 39.4 & $5.79 \times 10^{-19}$ & 0.860 \\
Air, $\lambda=0.25$ & 45.9 & $6.73 \times 10^{-19}$ & 1.000 \\
$\mathrm{O}_{2}, \lambda=0.21$ & 48.8 & $7.16 \times 10^{-19}$ & 1.064 \\
\hline
\end{tabular}

then concluded that the operation of constant $V$ and varying $\omega$, on the other hand, could better maintain the DMA-APM resolution because $m \omega^{2}$ can be constant under constant $V$. However, this ideal operation protocol is less employed for the DMA-APM system, mainly due to the practical impediment of quickly and accurately scanning $\omega$ over a range. Therefore, the common constant $\omega$ operation is investigated here.

As shown in Table 3, when determining the particle mass for $100 \mathrm{~nm}$ PSL spheres, respective differences of $-14 \%$ and $+6 \%$ are observed when $\mathrm{CO}_{2}$ and $\mathrm{O}_{2}$ are used as carrier gases in the APM. However, in Eq. (4), $S_{\mathrm{c}}$ is independent 

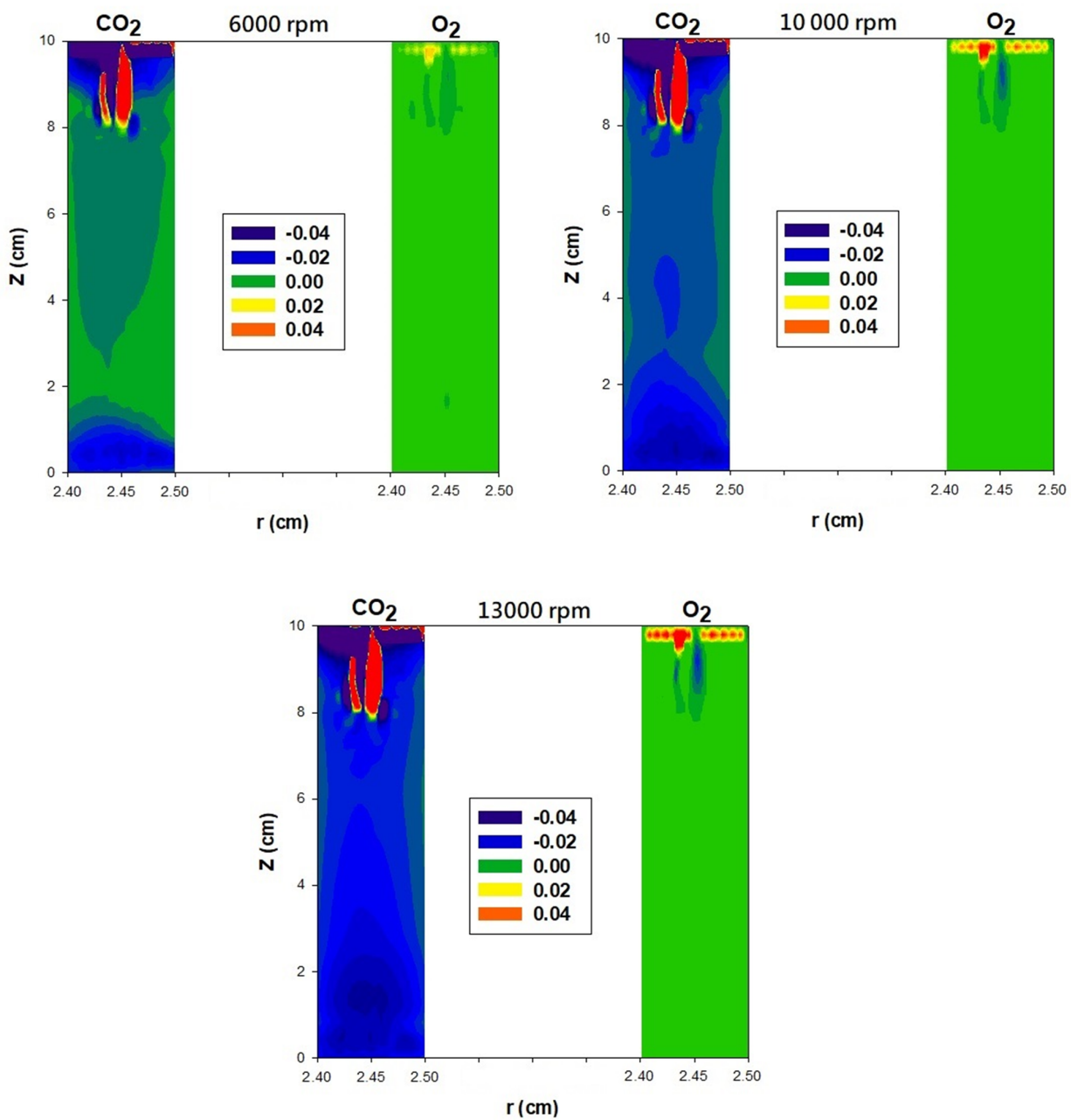

Figure 7. Computational fluid dynamic-simulated $\mathrm{CO}_{2}$ and $\mathrm{O}_{2}$ flow fields of APM at various rotation speeds.

of gas properties, and thus $S_{\mathrm{c}}$ or the peak voltage of the $C_{\mathrm{N}}-$ $V$ spectrum should remain unchanged if identical values of $\omega$ are applied in the APM for various carrier gases. The current experimental results demonstrate that the classification ability of APM is dependent on the carrier gas and influenced by gas viscosity. There could be changes in the flow field within the APM when $\mathrm{CO}_{2}$ and $\mathrm{O}_{2}$ are used, and radial acceleration may not be $r_{\mathrm{c}} \omega^{2}$, as used in Eq. (4). Therefore, to determine the exact centrifugal force for $S_{\mathrm{c}}$, further research is required to investigate the velocity profile of particles in an angular direction. In addition, when comparing air and $\mathrm{O}_{2}$, a broader $C_{\mathrm{N}}-V$ spectrum with weaker penetration was observed for $\mathrm{CO}_{2}$; this could be attributed to the higher diffusivity resulting from the lower gas viscosity of $\mathrm{CO}_{2}$ (StokesEinstein equation: $D=k_{B} T / 3 \pi \mu d_{p}$ ). Based on the results, on-site calibration of the APM's classification performance is strongly recommended.

\section{Conclusions}

In this study, the effects of temperature (" $T$ " hereafter), pressure (" $P$ " hereafter), and gas viscosity (" $\mu$ " hereafter) on the performance of the APM were evaluated analytically and experimentally. The analytical results revealed that the APM's detection limit can be lowered simply by increasing the $C_{\mathrm{c}} / \mu$ ratio without modifying the hardware of the APM or its classifying resolution. Under a constant $\lambda$ and fixed $T$ and $P$, the use of a low-molecular-weight carrier gas such as $\mathrm{H}_{2}$ or He can lower the mass detection limit to approximately $<10^{-2} \mathrm{fg}$. Similarly, a reduction in operating $P$ lowers the detection limit or improves the resolution. Under these circumstances, the effects of $T$ on the APM's detection limit are relatively minor.

Our experimental results under constant $\lambda$ or $\omega$ values reveal that the use of a carrier gas other than air reduces accuracy. Specifically, a carrier gas with a lower $\mu$ than air such as $\mathrm{CO}_{2}$ yields an underestimation of mass, whereas one with a higher $\mu$ such as $\mathrm{O}_{2}$ yields an overestimation. A subse- 
quent flow field simulation revealed variations in flow velocity in an angular direction at the APM's inlet and outlet when air and other carrier gases were used. The flow velocity decreased with $\mu$ but increased with $\omega$. Thus, the effects of $T$ are expected to affect the APM's performance, and changes in $\mu$, density, and diffusivity in the carrier gas likely alter the radial acceleration of flow in the APM; however, further research is recommended for these aspects.

Data availability. Data are available upon request by contacting the corresponding author.

Author contributions. TCH and LHY designed the research; YCT and PKC performed the research and analysed the data; and $\mathrm{TCH}$, LHY and YCT wrote the paper.

Competing interests. The authors declare that they have no conflict of interest.

Acknowledgements. The authors are grateful for the financial support provided by the National Science Council, Taiwan (NSC102-2221-E-008-004-MY3). The manuscript was edited by Wallace Academic Editing.

Edited by: Mingjin Tang

Reviewed by: three anonymous referees

\section{References}

Allen, M. D. and Raabe, O. G.: Slip Correction Measurements of Spherical Solid Aerosol Particles in an Improved Millikan Apparatus, Aerosol Sci. Technol., 4, 269-286, 1985.

Bau, S., Bémer, D., Grippari, F., Appert-Collin, J.-C., and Thomas, D.: Determining the effective density of airborne nanoparticles using multiple charging correction in a tandem DMA/ELPI setup, J. Nanopart. Res., 16, 1-13, 2014.

Broday, D. M. and Rosenzweig, R.: Deposition of fractal-like soot aggregates in the human respiratory tract, J. Aerosol Sci., 42, 372-386, 2011.

Chuang, H.-C., Hsiao, T.-C., Wang, S.-H., Tsay, S.-C., and Lin, N.H.: Characterization of Particulate Matter Profiling and Alveolar Deposition from Biomass Burning in Northern Thailand: The 7SEAS Study, Aerosol Air Qual. Res., 16, 2581-2602, 2016.

DeCarlo, P. F., Slowik, J. G., Worsnop, D. R., Davidovits, P., and Jimenez, J. L.: Particle Morphology and Density Characterization by Combined Mobility and Aerodynamic Diameter Measurements. Part 1: Theory, Aerosol Sci. Technol., 38, 1185-1205, 2004.

Ehara, K., Hagwood, C., and Coakley, K. J.: Novel method to classify aerosol particles according to their mass-to-charge ratio-Aerosol particle mass analyser, J. Aerosol Sci., 27, 217234, 1996.
Emery, M.: Theoretical Analysis of Data from DMA-APM System, Masters Thesis, University of Minnesota, 2005.

Haddrell, A. E., Davies, J. F., and Reid, J. P.: Dynamics of Particle Size on Inhalation of Environmental Aerosol and Impact on Deposition Fraction, Environ. Sci. Technol., 49, 14512-14521, https://doi.org/10.1021/acs.est.5b01930, 2015.

Hand, J. L. and Kreidenweis, S. M.: A New Method for Retrieving Particle Refractive Index and Effective Density from Aerosol Size Distribution Data, Aerosol Sci. Tech., 36, 1012-1026, 2002.

He, C., Liou, K.-N., Takano, Y., Zhang, R., Levy Zamora, M., Yang, P., Li, Q., and Leung, L. R.: Variation of the radiative properties during black carbon aging: theoretical and experimental intercomparison, Atmos. Chem. Phys., 15, 11967-11980, https://doi.org/10.5194/acp-15-11967-2015, 2015.

Karg, E., Dua, S. K., and Ferron, G. A.: Performance of a differential mobility analyzer at different gas compositions, J. Aerosol Sci., 23, Supplement 1, 389-392, 1992.

Kelly, W. P. and McMurry, P. H.: Measurement of Particle Density by Inertial Classification of Differential Mobility AnalyzerGenerated Monodisperse Aerosols, Aerosol Sci. Tech., 17, 199212, 1992.

Kulkarni, P., Baron, P. A., and Willeke, K.: Aerosol measurement: principles, techniques, and applications, John Wiley \& Sons, 2011.

Kuwata, M.: Particle Classification by the Tandem Differential Mobility Analyzer-Particle Mass Analyzer System, Aerosol Sci. Tech., 49, 508-520, 2015.

Kuwata, M. and Kondo, Y.: Measurements of particle masses of inorganic salt particles for calibration of cloud condensation nuclei counters, Atmos. Chem. Phys., 9, 5921-5932, https://doi.org/10.5194/acp-9-5921-2009, 2009.

Kuwata, M., Zorn, S. R., and Martin, S. T.: Using elemental ratios to predict the density of organic material composed of carbon, hydrogen, and oxygen, Environ. Sci. Technol., 46, 787-794, 2011.

Lall, A. A., Rong, W., Mädler, L., and Friedlander, S. K.: Nanoparticle aggregate volume determination by electrical mobility analysis: Test of idealized aggregate theory using aerosol particle mass analyzer measurements, J. Aerosol Sci., 39, 403-417, 2008.

Lall, A. A., Ma, X., Guha, S., Mulholland, G. W., and Zachariah, M. R.: Online Nanoparticle Mass Measurement by Combined Aerosol Particle Mass Analyzer and Differential Mobility Analyzer: Comparison of Theory and Measurements, Aerosol Sci. Tech., 43, 1075-1083, 2009.

Leskinen, J., Ihalainen, M., Torvela, T., Kortelainen, M., Lamberg, H., Tiitta, P., Jakobi, G., Grigonyte, J., Joutsensaari, J., Sippula, O., Tissari, J., Virtanen, A., Zimmermann, R., and Jokiniemi, J.: Effective Density and Morphology of Particles Emitted from Small-Scale Combustion of Various Wood Fuels, Environ. Sci. Technol., 48, 13298-13306, 2014.

Liu, F., Wong, C., Snelling, D. R., and Smallwood, G. J.: Investigation of Absorption and Scattering Properties of Soot Aggregates of Different Fractal Dimension at 532 nm Using RDG and GMM, Aerosol Sci. Tech., 47, 1393-1405, 2013.

Maricq, M. M., Podsiadlik, D. H., and Chase, R. E.: Size Distributions of Motor Vehicle Exhaust PM: A Comparison Between ELPI and SMPS Measurements, Aerosol Sci. Tech., 33, 239260, 2000. 
Marlow, W. H., Reist, P. C., and Dwiggins, G. A.: Aspects of the performance of the electrical aerosol analyzer under nonideal conditions, J. Aerosol Sci., 7, 457-462, 1976.

McMurry, P. H., Wang, X., Park, K., and Ehara, K.: The Relationship between Mass and Mobility for Atmospheric Particles: A New Technique for Measuring Particle Density, Aerosol Sci. Tech., 36, 227-238, 2002.

Myojo, T., Takaya, M., and Ono-Ogasawara, M.: DMA as a Gas Converter from Aerosol to "Argonsol" for Real-Time Chemical Analysis Using ICP-MS, Aerosol Sci. Tech., 36, 76-83, 2002.

Ogren, J. A.: On the operation of the electrical aerosol analyzer at reduced pressures, J. Aerosol Sci., 11, 427-434, 1980.

Park, K., Cao, F., Kittelson, D. B., and McMurry, P. H.: Relationship between Particle Mass and Mobility for Diesel Exhaust Particles, Environ. Sci. Technol., 37, 577-583, 2003.

Park, K., Kittelson, D., Zachariah, M., and McMurry, P.: Measurement of Inherent Material Density of Nanoparticle Agglomerates, J. Nanopart. Res., 6, 267-272, 2004a.

Park, K., Kittelson, D. B., and McMurry, P. H.: Structural Properties of Diesel Exhaust Particles Measured by Transmission Electron Microscopy (TEM): Relationships to Particle Mass and Mobility, Aerosol Sci. Tech., 38, 881-889, 2004b.

Rissler, J., Nordin, E. Z., Eriksson, A. C., Nilsson, P. T., Frosch, M., Sporre, M. K., Wierzbicka, A., Svenningsson, B., Löndahl, J., Messing, M. E., Sjogren, S., Hemmingsen, J. G., Loft, S., Pagels, J. H., and Swietlicki, E.: Effective Density and Mixing State of Aerosol Particles in a Near-Traffic Urban Environment, Environ. Sci. Technol., 48, 6300-6308, 2014.

Ristimäki, J., Virtanen, A., Marjamäki, M., Rostedt, A., and Keskinen, J.: On-line measurement of size distribution and effective density of submicron aerosol particles, J. Aerosol Sci., 33, 15411557, 2002.

Salma, I., Balásházy, I., Winkler-Heil, R., Hofmann, W., and Záray, G.: Effect of particle mass size distribution on the deposition of aerosols in the human respiratory system, J. Aerosol Sci., 33, 119-132, 2002.

Scheckman, J. H., McMurry, P. H., and Pratsinis, S. E.: Rapid Characterization of Agglomerate Aerosols by In Situ Mass-Mobility Measurements, Langmuir, 25, 8248-8254, 2009.
Schleicher, B., Künzel, S., and Burtscher, H.: Insitu measurement of size and density of submicron aerosol particles, J. Appl. Phys., 78, 4416-4422, 1995.

Schmid, O., Trueblood, M. B., Gregg, N., Hagen, D. E., and Whitefield, P. D.: Sizing of Aerosol in Gases Other Than Air Using a Differential Mobility Analyzer, Aerosol Sci. Tech., 36, 351-360, 2002.

Shi, Y., Ji, Y., Sun, H., Hui, F., Hu, J., Wu, Y., Fang, J., Lin, H., Wang, J., and Duan, H.: Nanoscale characterization of $\mathrm{PM}_{2.5}$ airborne pollutants reveals high adhesiveness and aggregation capability of soot particles, Sci. Rep.-UK, 5, 1123,2 https://doi.org/10.1038/srep1123, 2015.

Skillas, G., Künzel, S., Burtscher, H., Baltensperger, U., and Siegmann, K.: High fractal-like dimension of diesel soot agglomerates, J. Aerosol Sci., 29, 411-419, 1998.

Symonds, J. P. R., Reavell, K. S. J., Olfert, J. S., Campbell, B. W., and Swift, S. J.: Diesel soot mass calculation in real-time with a differential mobility spectrometer, J. Aerosol Sci., 38, 52-68, 2007.

Tajima, N., Fukushima, N., Ehara, K., and Sakurai, H.: Mass Range and Optimized Operation of the Aerosol Particle Mass Analyzer, Aerosol Sci. Tech., 45, 196-214, 2011.

Tajima, N., Sakurai, H., Fukushima, N., and Ehara, K.: Design Considerations and Performance Evaluation of a Compact Aerosol Particle Mass Analyzer, Aerosol Sci. Tech., 47, 1152-1162, 2013.

Van Gulijk, C., Marijnissen, J. C. M., Makkee, M., Moulijn, J. A., and Schmidt-Ott, A.: Measuring diesel soot with a scanning mobility particle sizer and an electrical low-pressure impactor: performance assessment with a model for fractal-like agglomerates, J. Aerosol Sci., 35, 633-655, 2004.

Virtanen, A., Ristimäki, J., and Keskinen, J.: Method for Measuring Effective Density and Fractal Dimension of Aerosol Agglomerates, Aerosol Sci. Tech., 38, 437-446, 2004. 